\title{
Diagnóstico microbiológico y molecular de bacterias cariogénicas en mujeres embarazadas de la Región de La Araucanía, Chile
}

\author{
Christian L. Herrera G., Patricio Pantoja F., Tomás de La Maza De la M., \\ Antonio Sanhueza C. y Luis A. Salazar N.
}

\begin{abstract}
Universidad de La Frontera,
Temuco, Chile

Facultad de Medicina

Departamento de Ciencias

Preclínicas (CLHG)

Estudiante de Odontología (PPF, TDLM)

Departamento de Ciencias Básicas

(LASN)

Facultad de Ingeniería, Ciencias y

Administración. Departamento de

Matemática y Estadística (ASC)

Financiamiento: Proyecto DIUFRO EP

120335, Dirección de Investigación y

Desarrollo, Universidad de La

Frontera y Programa "Aplicación

Sistema Financiamiento de Tesis Pro

Desarrollo IX Región-FNDR", año

2004, Código BIP 20167359-0.

Recibido: 25 de noviembre de 2006

Aceptado: 16 de abril de 2007
\end{abstract}

Correspondencia a: Luis A. Salazar Navarrete |salazar@ufro.cl

\section{Introducción}

E n Chile, la situación de la salud dentaria es bastante estremecedora. Si bien, no existen estudios nacionales en relación a la caries dental en el adulto, el año 1995 se determinó que 100\% de los adultos de nivel socioeconómico bajo y medio-bajo, residentes en la Región Metropolitana (RM) presentaban caries con índices COPD (Dientes Cariados, Obturados y Perdidos) superiores a 25 en individuos mayores de 35 años $^{1}$. En el año 1999, un estudio también realizado en la RM, comprobó que $98 \%$ de las mujeres embarazadas de nivel socioeconómico bajo presentaba caries ${ }^{2}$ y la encuesta nacional de salud efectuada por el MINSAL durante el año 2003, señala que más de $70 \%$ de la población manifiesta tener su dentadura incompleta, a $90 \%$ de los individuos de nivel socioeconómico bajo le faltan piezas dentales y $66 \%$ de la población general tiene caries ${ }^{3}$.

En el caso de los niños, estudios realizados durante los años 1996 y 1999, mostraron la presencia de altos índices de caries, con una prevalencia nacional de caries dentales de $84 \%$ en escolares de 6 a 8 y 12 años. En la Región de la Araucanía, la situación es aún más dramática, ya que la prevalencia de caries para los niños de 6 a 8 años es de $96,7 \%$ y para los de 12 años es de $97,8 \%$, siendo ambas prevalencias las más altas del país ${ }^{4}$.

Estudios clínicos han demostrado que los tejidos orales pueden ser afectados por el embarazo. Si bien está ampliamente difundida la creencia que la gestación es perjudicial para la dentadura, los efectos del embarazo en la iniciación o progresión de las caries no son claros. Existen evidencias de que los dientes no se ablandan y que los niveles de calcio u otros minerales no disminuyen en forma significativa; es principalmente el ambiente del diente el que se ve afectado, al aumentar el número de microorganismos cariogénicos, coincidentemente, con la disminución del $\mathrm{pH}$ y de la actividad tampón de la saliva durante la gestación ${ }^{5}$. Es difícil estimar la relación entre el aumento de caries y embarazo; sin embargo, Banoczy y cols, encontraron que las mujeres con hijos tienen un COPD más alto que las mujeres sin hijos ${ }^{6}$.

Considerando la compleja realidad de la salud oral en Chile, específicamente la alta prevalencia de caries y la no existencia de antecedentes sobre esta patología en mujeres embarazadas de regiones distintas a la 
$\mathrm{RM}$, se hace necesario conocer cuál es la realidad de la salud dentaria de estas mujeres. Esto, no sólo considerando la trascendencia que tiene para ella misma, sino que, más importante aún, el hecho de que la calidad de la salud bucal de los niños, tiene relación con la salud bucal de sus madres; se ha demostrado que la principal fuente de adquisición y transmisión de las bacterias con potencial cariogénico en niños, es a partir de la saliva de sus propias madres ${ }^{7,8}$.

Alrededor de 300 especies bacterianas han sido asociadas con la placa dental, pero sólo la presencia de Streptococcus del grupo mutans ha sido relacionada con la formación de caries dental en humanos, considerándose como el principal agente productor de caries, y dentro de este grupo, Streptococcus mutans (Streptococcus grupo mutans especie mutans) y Streptococcus sobrinus (Streptococcus grupo mutans especie sobrinus) son los que aparecen con mayor frecuencia ${ }^{9,10}$.

En la actualidad, los métodos de detección e identificación fenotípica de bacterias cariogénicas y, específicamente, de Streptococcus grupo mutans, utilizan pruebas como: manitol, resistencia a bacitracina, arginina dehidrolasa, inulina, esculina y rafinosa, entre otros, lo que los hace bastante laboriosos y exten$\operatorname{sos}^{11}$. Como consecuencia de esto, se han comenzado a aplicar técnicas de biología molecular basadas en la reacción de polimerasa en cadena (RPC), técnica que permite obtener resultados altamente sensibles y específicos, con rapidez y de forma relativamente senci$11 a^{12-14}$.

La técnica de RPC tiene por finalidad amplificar segmentos específicos del ADN de una célula, lo que permite su detección e identificación. En el caso de Streptococcus del grupo mutans se han utilizado variados genes específicos para las diferentes especies pertenecientes a este grupo; algunos ejemplos son: gtfB, groESL, wapA y spaP $P^{12,15-17}$.

Por lo señalado anteriormente, esta investigación busca dar a conocer el grado y la frecuencia de infección por bacterias responsables de la producción de caries en mujeres embarazadas, grupo de riesgo para el desarrollo de esta enfermedad, mediante análisis microbiológico y molecular, en una de las regiones de Chile donde la prevalencia de caries es más elevada.

\section{Material y Método}

Pacientes y muestras clínicas: Se estudiaron 51 mujeres embarazadas, de nivel socioeconómico bajo, edades entre 15 y 40 años, con un promedio de edad de $27 \pm 8$ años, y una edad gestacional de $14 \pm 8$ semanas, de las cuales 17 tenían ascendencia mapuche ${ }^{1}$ *
El promedio de edad de este grupo fue de $25 \pm 8$ años, y la edad gestacional de $18 \pm 8$ semanas. Todas las pacientes provenían del Consultorio de Atención Secundaria Miraflores de la ciudad de Temuco (capital de la Región de La Araucanía, Chile).

Criterios de exclusión: Se excluyeron las mujeres que estuvieran haciendo uso de terapia antimicrobiana, tanto al momento del estudio como dos meses previos a éste; que se encontraran en tratamiento con algún otro medicamento; las que presentaban signos clínicos de xerostomia o la presencia de enfermedades metabólicas, como diabetes mellitus o diabetes gestacional.

El estudio contó con la aprobación del Comité de Ética de la Facultad de Medicina, Universidad de La Frontera. Todas las participantes firmaron voluntariamente un consentimiento informado.

Análisis clínico: Las condiciones de sus dentaduras fueron evaluadas mediante un examen clínico oral, en el que se obtuvo el índice COPD, por medio de una ficha clínica que tuvo la función de contener la información general del paciente: datos personales, antecedentes mórbidos y médicos, junto con los antecedentes odontológicos personales. Además, contiene un detalle del examen intraoral realizado y el índice COPD arrojado.

Análisis microbiológico: La detección de los microorganismos cariogénicos fue realizada mediante el método descrito por Linossier y cols ${ }^{18}$. De acuerdo con el resultado de los recuentos, las pacientes se clasificaron dentro de tres rangos de riesgo cariogénico: bajo, medio y alto.

Análisis molecular: Para la detección e identificación de las bacterias $S$. mutans y $S$. sobrinus se amplificó un fragmento específico del ADN cromosomal de estas bacterias, mediante la técnica de RPC, utilizando como templado saliva. Las secuencias de los partidores usados, el tamaño de los fragmentos amplificados, la posición en el gen y $\mathrm{T}^{\mathrm{o}}$ de hibridación son descritos en la Tabla 1 .

Para evaluar los productos de la RPC, estos se tiñeron con bromuro de etídio y se realizó electroforesis en gel de agarosa, visualizando los productos de la $\mathrm{RPC}$ en un transiluminador UV.

Análisis estadístico: Los resultados fueron analizados mediante el programa estadístico SigmaStat para

\footnotetext{
*Definido por el criterio de dos apellidos mapuches y el reconocimiento por la
} paciente de su pertenencia a la etnia señalada. 
Tabla 1. Secuencia de los partidores utilizados para la detección e identificación de S. mutans y S. sobrinus en muestras de saliva por RPC

\begin{tabular}{llccc} 
Partidores & \multicolumn{1}{c}{ Secuencia } & $\begin{array}{c}\text { Localización } \\
\text { CADN }\end{array}$ & $\begin{array}{c}\text { To } \\
\text { hibridación }\end{array}$ & $\begin{array}{c}\text { Fragmento } \\
\text { (pb) }\end{array}$ \\
\hline SMUT5 & 5'-tgaaaccttgtctatctcctctttacc-3' & $1.760-1.783$ & 53 & 137 \\
SMUT3 & 5'-tcagtttccaaagggctctg-3' & $1.877-1.896$ & & \\
SSOB5 & 5'-gcagtctgacgatgcttctac-3' & $184-204$ & 50 & 198 \\
SSOB3 & 5'-ccgtcagtccaacaaataatc-3' & $361-381$ & & \\
\hline
\end{tabular}

La secuencia de nucleótidos fue obtenida desde el GenBank, Número de acceso AE014854.1 para Streptococcus mutans y NAB083137.1 para Streptococcus sobrinus.
Figura 1. Recuentos de Streptococcus grupo mutans (ufc/mL) en el grupo de mujeres embarazadas, según trimestre de gestación. Número de muestras entre paréntesis.
Tabla 2. Grupos de riesgo clasificados de acuerdo al recuento de ufc/mL de Streptococcus del grupo mutans, obtenido por método semicuantitativo

$$
\text { (Linoscreen } \circledast \text { ) }
$$

\begin{tabular}{|llcc|} 
ufc/mL & Riesgo & $\mathbf{n}$ & $\mathbf{\%}$ \\
\hline $10.000-50.000$ & Bajo & 9 & 17,6 \\
\hline $100.000-250.000$ & Medio & 6 & 11,8 \\
\hline $500.000-1.000 .000$ & Alto & 36 & 70,6 \\
\hline Total & & 51 & 100 \\
\hline n, número de individuos & & & \\
\hline
\end{tabular}

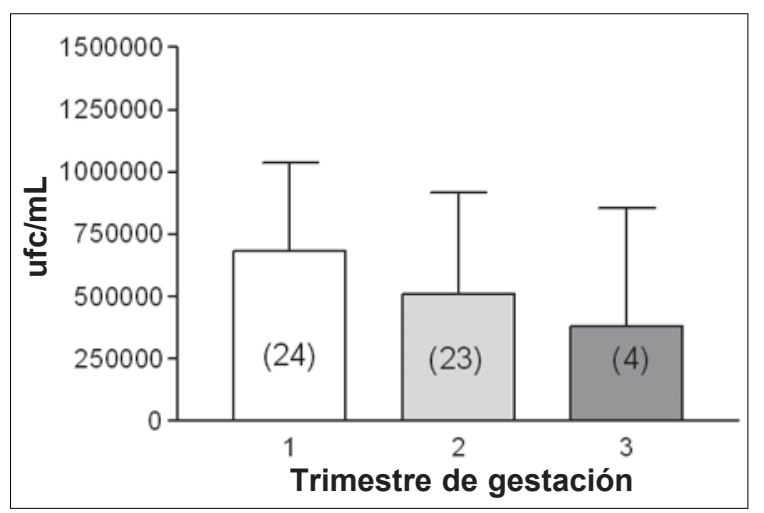

Tabla 3. Comparación de los niveles de riesgo cariogénico en relación al trimestre de gestación

\begin{tabular}{|lcccccc|}
\hline Riesgo & \multicolumn{2}{c}{ Bajo } & \multicolumn{2}{c}{ Medio } & \multicolumn{2}{c|}{ Alto } \\
& n & \% & n & \% & n & \% \\
\hline Trimestre 1 & 2 & 8,3 & 2 & 8,3 & 20 & 83,3 \\
\hline Trimestre 2 & 5 & 21,7 & 4 & 17,4 & 14 & 60,9 \\
\hline Trimestre 3 & 2 & 50,0 & 0 & 0 & 2 & 50,0 \\
\hline n, número de individuos & & & & & \\
\hline
\end{tabular}

Windows, versión 2.0 (San Rafael, CA, EE.UU.). Las variables continuas fueron evaluadas en forma descriptiva. Se procedió al cálculo de promedios, desviaciones estándar y la observación de valores máximos y mínimos. La asociación entre las diferentes variables fue evaluada mediante los tests $t$ de Student o ANOVA. El nivel de significancia utilizado fue $\mathrm{p}<0,05$.

\section{Resultados}

El análisis clínico mostró que las pacientes estudiadas tenían un COPD de 10,2 $\pm 4,7$, lo que indica una historia de caries cercana al $30 \%$ de la dentadura. Además, $100 \%$ de las mujeres embarazadas presentaba gingivitis.

Los resultados de los recuentos microbiológicos para $S$. grupo mutans muestran que la totalidad de las mujeres estudiadas presentan estas bacterias en su cavidad oral ( $>10.000 \mathrm{ufc} / \mathrm{mL})$. En la Tabla 2 se presenta la clasificación del riesgo de acuerdo al recuento de colonias de $S$. grupo mutans en saliva.

Al evaluar el grupo de mujeres según trimestre de gestación y relacionarlo con los recuentos de colonias, llama la atención que las mujeres que se encontraban en el primer trimestre de embarazo presentaron valores de ufc/mL mayores que aquellas que se encontraban en el segundo y tercer trimestre de gestación. Sin embargo, esta diferencia no fue significativa $(\mathrm{p}=0,184$, Figura 1$)$.

Al analizar los resultados de la clasificación de riesgo según trimestre de gestación, $83,3 \%$ de las mujeres que se encontraban en el primer trimestre $(<12$ semanas) presentaron un nivel de riesgo alto, a diferencia de las mujeres que se encontraban en el segundo y tercer trimestre, en las que se observó 60,9 y 50\%, respectivamente (Tabla 3). Sin embargo, las diferencias no fueron significativas $(\mathrm{p}=0,185)$.

Adicionalmente, al comparar los recuentos de $S$. grupo mutans según etnia, se observó similitud en el recuento promedio de colonias entre mujeres de ascendencia Mapuche y no Mapuche. Además, al comparar los índices COPD de ambos grupos, éstos también se mostraron similares, y no presentaron diferencias estadísticamente significativas (Tabla 4).

El resultado de la detección e identificación de Streptococcus cariogénicos por técnica de RPC se puede observar en la Figura 2, donde se muestran los productos de amplificación que indican la presencia de $S$. mutans (137 pb) y S. sobrinus (198 pb).

La frecuencia de $S$. mutans en el grupo de estudio fue de $92,1 \%(47 / 51)$ y de $S$. sobrinus fue de $1,9 \%(1 / 51)$. La presencia de ambas bacterias en conjunto, fue detectada en $1,9 \%$ de las muestras (1/51). 


\begin{tabular}{|c|c|c|c|c|c|c|}
\hline & $\mathbf{n}$ & Recuento ufc/mL* & Bajo & $\begin{array}{l}\text { de riesg } \\
\text { Medio }\end{array}$ & Alto & COPD** \\
\hline Mapuches & 17 & $572.353 \pm 446.955$ & 29,4 & 5,9 & 64,7 & $12,1 \pm 4,9$ \\
\hline No mapuches & 34 & $583.529 \pm 372.468$ & 11,8 & 14,7 & 73,5 & $9,3 \pm 4,3$ \\
\hline
\end{tabular}

Las muestras negativas $(4 / 51)$ para la técnica de RPC y que presentaban un recuento positivo, podrían explicarse porque existen otras especies dentro del grupo mutans que no son posibles de detectar con los partidores usados, ya que estos tienen una alta especificidad.

\section{Discusión}

Desde varias décadas, la caries dental ha sido reconocida como una enfermedad infecto-contagiosa originada por un grupo de bacterias conocidas como Streptococcus grupo mutans ${ }^{19}$.

La infancia temprana puede ser el período más importante para la salud dental futura, ya que mientras antes se produzca la infección por Streptococcus del grupo mutans, mayor es el riesgo de desarrollar caries $^{20}$. Es aquí donde cobra mayor importancia el estudio de la microbiota oral en la mujer gestante, ya que los cambios en el embarazo pueden predisponer al desarrollo de caries al aumentar los recuentos de bacterias cariogénicas y aumentar el riesgo de infección de sus hijos, con el consecuente riesgo de éstos para desarrollar caries 5 .

El análisis clínico mostró un índice COPD de $10,2 \pm 4,7$. En Chile, sólo existe una publicación anterior en relación a recuentos de Streptococcus grupo mutans en mujeres embarazadas ${ }^{2}$, donde se observó un índice COPD de 15,8 $\pm 5,4$; resultado similar al obtenido en un estudio realizado en la ciudad de Bauru $(\text { Brasil })^{21}$, que encontró un índice COPD de 14.

La prevalencia de Streptococcus grupo mutans en nuestro grupo de estudio fue de $100 \%$, lo que es concordante con lo encontrado en la literatura médi$\mathrm{ca}^{2,21}$. Es importante señalar que $70,6 \%$ del grupo en estudio presentó recuentos superiores a $500.000 \mathrm{ufc} /$ $\mathrm{mL}$, lo que indica un alto riesgo para el desarrollo de caries, de acuerdo con el método microbiológico utilizado.

Los recuentos de $S$. grupo mutans no tuvieron dife-

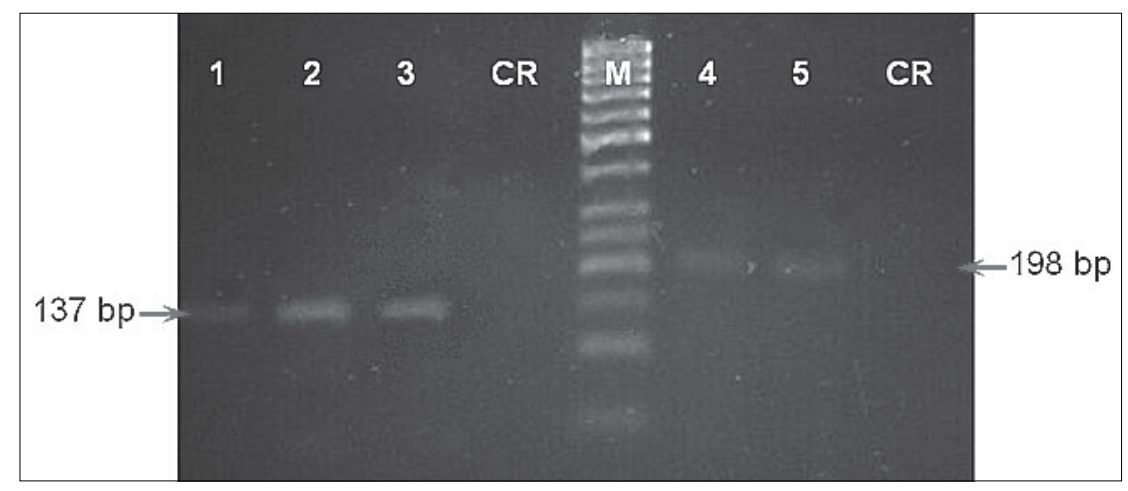

Figura 2. Electroforesis en gel de agarosa al $2 \%$ teñido con bromuro de etidio, que muestra los fragmentos indicadores de la presencia de Streptococcus mutans (carriles 1, 2 y 3) y Streptococcus sobrinus (carriles 4 y 5). M, marcador de tamaño molecular de ADN (50 pb) y CR, control de reactivos.

rencia significativa de acuerdo con el trimestre de embarazo. Resultado similar al obtenido por Villagrán y cols. $^{2}$, en un estudio realizado en 174 mujeres embarazadas de la RM.

Los resultados del análisis molecular, indican que la presencia de la especie $S$. mutans es la de mayor frecuencia, lo que concuerda con lo observado por otros autores $^{22-25}$. Por otro lado, $S$. sobrinus se encuentra en una proporción muy inferior. Respecto del análisis de esta especie bacteriana, existen diferencias notables entre las frecuencias encontradas por diversos autores $^{12,22-25}$. Sólo una de las pacientes presentó asociación entre $S$. mutans / S. sobrinus, hecho muy relevante, ya que está demostrado que esta asociación se presenta en individuos con índices de caries superiores a los que presentan sólo $S$. mutans ${ }^{26}$.

La existencia de muestras negativas mediante la técnica de RPC, puede deberse a que existen otras especies del grupo Streptococcus mutans tales como, S. rattus, S. cricetus, S. ferus, S. downei y S. macacae, que no pueden ser identificadas con los partidores utilizados, lo que confirma la alta especificidad de la técnica de RPC.

Al clasificar el grupo en estudio, en mapuches y no 
mapuches, no se observaron diferencias significativas en los parámetros estudiados (COPD, recuento bacteriológico y análisis molecular).

Este estudio destaca la importancia del control odontológico pre y post-natal, tanto desde el punto de vista de la prevención como del tratamiento, ya que está comprobado que estas actividades en la mujer embarazada tienen importantes efectos, reduciendo los niveles de $S$. grupo mutans y de caries en forma significativa tanto en ella como en su descenden$\mathrm{cia}^{27-30}$.

Es importante destacar también, que la técnica de RPC es utilizada por primera vez en nuestro país para la identificación de bacterias cariogénicas en mujeres embarazadas y que, por su rapidez, alta especificidad y sensibilidad, es una alternativa válida, tanto al momento de desarrollar estudios epidemiológicos como para la evaluación y diagnóstico de pacientes. Si bien esta técnica permite la identificación, y no la cuantificación de el o los agentes etiológicos, su aplicación puede ser de gran importancia al momento de seleccionar un esquema de tratamiento, debido a que no todas las especies del grupo mutans poseen el mismo potencial cariogénico.

\section{Conclusiones}

Las mujeres embarazadas estudiadas se encuentran con un alto riesgo de desarrollar caries ya que, la totalidad de ellas, se encuentran infectadas por Streptococcus grupo mutans y tres cuartas partes del grupo estudiado presentan un elevado riesgo cariogénico, desde el punto de vista microbiológico. Estos antecedentes indican que es necesario tomar medidas tendientes a mejorar la salud bucal de las mujeres durante la gestación, con el objetivo de prevenir el desarrollo de caries en ellas y la transmisión temprana de bacterias cariogénicas a sus hijos.

\section{Resumen}

La caries dental es una enfermedad infecciosa, en la cual Streptococcus del grupo mutans, son los principales protagonistas. Aunque está ampliamente difundida la creencia de que el embarazo es perjudicial para la salud dental, el real efecto de esta condición sobre el desarrollo de caries aún no está claro. Considerando esta situación, el objetivo de nuestro trabajo fue evaluar los niveles de infección en mujeres embarazadas de la Región de La Araucanía (Chile) y diferenciar especies bacterianas con potencial cariogénico, de aquellas sin este potencial, mediante análisis bacteriológico y molecular. En el presente estudio fueron evaluadas 51 mujeres gestantes con edades que fluctuaron entre 15 y 40 años. Los resultados muestran que $100 \%$ de las mujeres se encuentran infectadas por Streptococcus del grupo mutans, de las cuales 70,6\% muestra un riesgo elevado de desarrollar caries (>500.000 ufc/ml). El análisis molecular mostró que las frecuencias de Streptococcus mutans y Streptococcus sobrinus fueron $92,1 \%$ y $1,9 \%$, respectivamente. En conclusión, nuestros datos sugieren que las mujeres embarazadas son un grupo de alto riesgo para el desarrollo de caries dental.

\section{Referencias}

1.- Gamonal J A, López N J, Aranda W. Periodontal conditions and treatment needs, by CPITN, in the 35-44 and 65-74 year-old population in Santiago, Chile. Int Dent J 1998; 48: 96-103.

2.- Villagrán E, Linossier A, Donoso E. Count of salivary Streptococci mutans in pregnant women of the metropolitan region of Chile: cross-sectional study. Rev Méd Chile 1999; 127: 165-70.

3.- MINSAL. Encuesta Nacional de Salud 2003. Santiago. Marzo 2004.

4.- Urbina T, Caro J P, Vicent M. Caries y fluorosis en niños de 6 a 8 años y 12 años Minsal, Chile. Departamento de Estadísticas e Información. 1996-1999.

5.- Laine M A. Effect of pregnancy on periodontal and dental health. Acta Odontol Scand 2002; 60: 257-64.

6.- Banoczy J, Orosz M, Gabris K, Nyarasdy I, Rigo $\mathrm{O}$, Schuder $\mathrm{L}$. Investigation on the correlation of pregnancy, caries and gingivitis (author's transl). Zahn Mund Kieferheilkd Zentralbl 1978; 66: 573-81.

7.- Lindquist B, Emilson C G. Colonization of Streptococcus mutans and Streptococcus sobrinus genotypes and caries development in children to mothers harboring both species. Caries Res 2004; 38: 95-103.

8.- Mattos-Graner R O, Li Y, Caufield P W, Duncan M, Smith D J. Genotypic diversity of mutans streptococci in Brazilian nursery children suggests horizontal transmission. J Clin Microbiol 2001; 39: 2313-6.

9.- Ajdic D, McShan W M, McLaughlin R E, Savic G, Chang J, Carson M B, et al. Genome sequence of Streptococcus mutans UA159, a cariogenic dental pathogen. Proc Natl Acad Sci 2002; 99: 14434-9.

10.- Loesche WJ. Role of Streptococcus mutans in human dental decay. Microbiol Rev 1986; 50: $353-80$.

11.- Liébana J. Microbiología Oral. $2^{\mathrm{a}}$ Edición 2002, McGraw-Hill Interamericana de España: Cap. 11 y 32.

12.- Igarashi T, Yamamoto A, Goto N. PCR for detection and identification of Streptococcus sobrinus. J Med Microbiol 2000; 49: 106974.

13.- Yano A, Kaneko N, Ida H, Yamaguchi T, Hanada N. Real-time PCR for quantification of Streptococcus mutans. FEMS Microbiol Lett 2002; 217: 23-30.

14.- Aguilera L, Estrada I. Detección de una secuencia del gene spaP de Streptococcus 
mutans en muestras de placa dental mediante reacción en cadena de la polimerasa. Revista ADM 2003; LX (5): 180-4.

15.- Nascimento M M, Hofling J F, Goncalves R B. Streptococcus mutans genotypes isolated from root and coronal caries. Caries Res 2004; 38: 454-63.

16.- Hung W C, Tsai J C, Hsueh P R, Chia J S, Teng L J. Species identification of mutans streptococci by groESL gene sequence. J Med Microbiol 2005; 54: 857-62.

17. - Zhu L, Kreth J, Cross S E, Gimzewsky J K, Shi W, Oi F. Functional characterization of cell-wall-associated protein WapA in Streptococcus mutans. Microbiology 2006; 152: 2395-2404.

18.- Linossier A, Vargas A, Zillmann G, Arriagada M, Rojas R, Villegas R. Streptococci mutans: a semi-quantitative method to assess the risk to oral infection in preschool Chilean children. Rev Méd Chile 2003; 131: 412-18.

19.- Balakrishnan M, Simmonds R S, Tagg J R. Dental caries is a preventable infectious disease. Aust Dent J 2000; 45: 235-45.

20.- Mattos-Graner R O, Correa M S, Latorre M R, Peres R C, Mayer M P. Mutans streptococci oral colonization in 12-30-month-old
Brazilian children over a one-year follow-up period. J Public Health Dent 2001; 61: 161-7.

21.- Torres S A, Rosa O, Akiyoshi N, Silveira A, Bretz W. Niveis de infecção de estreptococos do grupo mutans em gestantes. Rev Odontol Univ Sao Paulo. 1999; 13: 225-31.

22.- Hoshino T, Kawaguchi M, Shimizu N, Hoshino N, Ooshima T, Fujiwara T. PCR detection and identification of oral streptococci in saliva samples using $g t f$ genes. Diagn Microbiol Infect Dis 2004; 48 : 195-9.

23.- Oho T, Yamashita Y, Shimazaki Y, Kushiyama M, Koga T. Simple and rapid detection of Streptococcus mutans and Streptococcus sobrinus in human saliva by polymerase chain reaction. Oral Microbiol Immunol 2000; 15: 258-62.

24.- Sato T, Matsuyama J, Kumagai T, Mayanagi G, Yamaura M, Washio J, et al. Nested PCR for detection of mutans streptococci in dental plaque. Lett Appl Microbiol 2003; 37: $66-9$

25.- Okada M, Soda Y, Hayashi F, Doi T, Suzuki J, Miura K. PCR detection of Streptococcus mutans and $S$. sobrinus in dental plaque samples from Japanese pre-school children. J
Med Microbiol 2002; 51: 443-7.

26. - Hofling J F, Spolidorio D M, Pereira C V, Rosa E A R, Moreira D. Presença de Streptococcus mutans e Streptococcus sobrinus em escolares de diferentes classes sócio-econômicas e sua relação com a atividade cariogênica dessas populações. Rev Odontol Univ São Paulo 1999; 13: 173-80.

27.- Gunay H, Dmoch-Bockhorn K, Gunay Y, Geursen W. Effect on caries experience of a long-term preventive program for mothers and children starting during pregnancy. Clin Oral Investig 1998; 2: 137-42.

28. - Zanata R L, Navarro M F, Pereira J C, Franco E B, Lauris J R, Barbosa S H. Effect of caries preventive measures directed to expectant mothers on caries experience in their children. Braz Dent J 2003; 14: 75-81.

29.- Gómez S S, Weber A A, Emilson C G. A prospective study of a caries prevention program in pregnant women and their children five and six years of age. ASDC J Dent Child 2001; 68: 191-5, 152.

30.- Brambilla E, Felloni A, Gagliani M, Malerba A, García-Godoy F, Strohmenger L. Caries prevention during pregnancy: results of a 30-month study. J Am Dent Assoc 1998; 129: 871-7. 